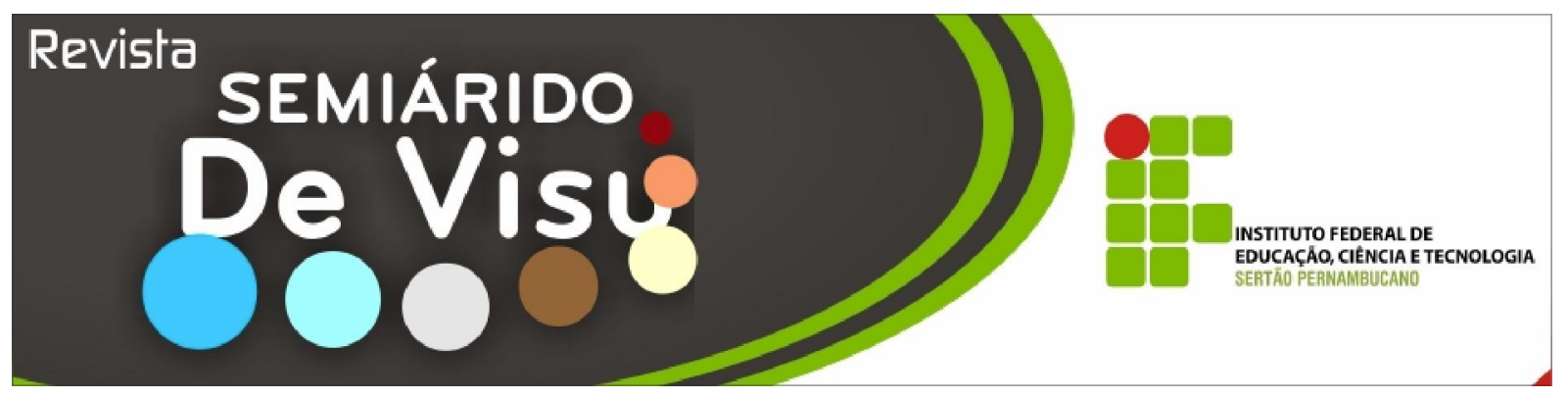

\title{
Composição florística do banco de sementes do solo da caatinga em perímetro irrigado de Petrolina - Pernambuco
}

\author{
Rosineide Gonçalves Parente ${ }^{1}$; Laise Guerra Barbosa ${ }^{1}$; Oafaela da Conceição Souza ${ }^{1}$; Flávia \\ Cartaxo Ramalho Vilar ${ }^{1}$. \\ ${ }^{1}$ Instituto Federal de Educação, Ciência e Tecnologia do Sertão Pernambucano. Campus Petrolina Zona Rural - \\ Rodovia BR 235 Km 22 - Projeto Senador Nilo Coelho (N4), Telefone/Fax (87)3862-3800, flavia.cartaxo@,ifsertao- \\ pe.edu.br.
}

\begin{abstract}
RESUMO: O conhecimento da densidade e composição florística do banco de sementes na caatinga é um instrumento primordial para identificar a riqueza das espécies herbáceas e sua regeneração no bioma, após o uso pela agricultura ou distúrbios antrópicos. Diante disto, foram selecionadas duas áreas formadas por remanescentes de caatinga, sendo um remanescente em bom estado de conservação (Área I) e outro com uma conservação regular (Área II), para estudar a composição florística do estrato herbáceo-subarbustivo do banco de sementes da caatinga, tentando evidenciar as alterações sofridas pelo uso da agricultura irrigada. Em cada uma das áreas selecionadas, durante a estação chuvosa foram plotadas 100 parcelas, como gabarito padronizador. Nas amostras coletadas procedeu-se ao levantamento florístico e a análise fitossociológica, mediante numeração e identificação dos indivíduos inseridos nas unidades amostrais. O Banco de Sementes do Solo (in situ), nas duas áreas estudadas, registrou um total de 2.199 indivíduos, distribuídos em 19 famílias, 54 gêneros e 71 espécies. O maior Índice de Diversidade Florística de Shannon-Weaver (H') foi encontrada na Área II com 3,23.
\end{abstract}

Palavras-chave: Fitossociologia, densidade, in situ.

\section{Floristic composition of the Caatinga Soil Seed Bank in irrigated Petrolina- Pernambuco}

\begin{abstract}
Knowledge of the density and the floristic compositon of seed banks in the caatinga is a major tool to identify the richness of herbaceous species and their regeneration in the biome after the use of agriculture or by human disturbances. Therefore, we selected two areas formed by remnants of caatinga, with a surplus in good condition (Area I) and another with regular maintenance (Area II) in order to study the floristic composition of the herbaceous and woody caatinga seed bank, trying to highlight the changes caused by the use of irrigated agriculture. In each of the selected areas 100 areas were plotted during the rainy season, as a template. Floristic and phytosociological analysises were made by numbering and identifying of the individuals in the sample units. The soil seed bank (in situ), in the two areas studied, recorded a total of 2199 individuals belonging to 19 families, 54 genera and 71 species. The largest Shannon-Weaver Floristic Diversity Index (H') was found in Area II with 3.23.
\end{abstract}

key words: Phytosociology, density, in situ. 
Rosineide Gonçalves Parente et. al

\section{Introdução}

$$
4 \begin{aligned}
& \text { agricultura irrigada na caatinga } \\
& \text { nordestina para atender a } \\
& \text { demanda da população por } \\
& \text { alimento, trouxe mudanças }
\end{aligned}
$$
desfavoráveis ao meio ambiente, modificando as características da vegetação em diferentes níveis.

O estrato herbáceo-subarbustivo da caatinga é fonte de alimento para os animais silvestres e domésticos, sendo amplamente utilizada na medicina popular, como lenha e no artesanato pelas comunidades locais. Pouco se conhece sobre o uso destes recursos naturais pelas comunidades locais, necessitando assim, catalogar urgentemente as espécies herbáceas, conhecer seus efeitos, suas propriedades e utilizações. O desconhecimento da diversidade florística e do potencial genético das espécies nativas da caatinga, bem como o estabelecimento e ocupação de Áreas deste bioma pela agricultura irrigada tem contribuído para sua exploração irracional e destruição.

O banco de sementes é uma reserva destas ou de propágulos vegetativos viáveis presentes no solo e que são capazes de recompor uma vegetação (Christoffoleti \& Caetano, 1998). É também um arquivo de informações das condições ambientais e práticas culturais anteriores, sendo fator importante de avaliação do potencial de infestação das plantas daninhas no presente e no futuro (Templeton \& Levin, 1979).

A identificação correta das espécies que compõem o estrato herbáceo/arbustivo do banco de sementes do solo, aliado ao estudo fitossociológico dessas comunidades, é uma maneira segura de identificar e quantificar sua diversidade. O conhecimento da densidade e composição florística do banco de sementes na caatinga é um instrumento primordial para identificar a riqueza das espécies herbáceas e sua regeneração no bioma, após o uso pela agricultura ou distúrbios antrópicos. Além disso, o conhecimento da utilização desses recursos naturais pelas comunidades locais permitirá avaliar de maneira qualitativa e quantitativa os impactos causados pela agricultura irrigada.

\section{Material e métodos}

\section{Descrição geral área de estudo}

Foram selecionadas duas áreas formadas por remanescentes de caatinga, sendo um remanescente em bom estado de conservação (Área I) e outro com uma conservação regular (Área II). Todas as áreas foram desmatadas e sofreram queimadas, depois foram exploradas pela agropecuária e em seguida mantidas em repouso por cerca de 10 anos.

\subsection{As áreas estudadas apresentaram os seguintes históricos de uso:}

1. Área I - Projeto Maria Tereza: caatinga arbóreo-arbustiva, localizada no distrito 22, com aproximadamente 36 hectares, em bom estado de conservação, sem corte raso há mais de 20 anos.

2. Área II - Projeto N-6-Sítio Asa Branca: em regular estado de conservação, estimada em cerca de 50 hectares, sendo 2 ha cultivados, 30 ha preservados e 18 ha atividades agropastoris.

\section{Estudo do Banco de Sementes do Solo - BSS}

\subsection{Banco de Sementes do Solo in situ (BSS in situ)}

Em cada uma das áreas selecionadas, durante a estação chuvosa foram plotadas 100 parcelas, com auxílio de um retângulo de madeira medindo $0,25 \mathrm{~m} \times 0,16 \mathrm{~m}$, com $0,03 \mathrm{~m}$ de altura, como gabarito padronizador o qual foi colocado sobre o solo aleatoriamente, de forma a cobrir toda a área amostrada, em seguida coletou-se todos os indivíduos contidos no seu interior.

As amostras coletadas foram colocadas em sacos numerados e em seguidas transportadas para o laboratório de Desenvolvimento Vegetal onde procedeu-se ao levantamento florístico, mediante numeração e identificação dos indivíduos inseridos nas unidades amostrais. A identificação botânica por comparação, utilizando os trabalhos de Kissmann \& Groth. (1991-2000). 
Rosineide Gonçalves Parente et. al

Os dados biométricos foram tomados e anotados em fichas de controle, para posterior elaboração de planilhas eletrônicas. Por ocasião dessa leitura, foi coletado material botânico para confecção de exsicatas e identificação das espécies em herbário.

\subsection{Estrutura Vegetacional}

Em cada área, foram analisados os seguintes parâmetros fitossociológicos do banco de semente (in situ): Densidade Relativa (DR), Frequências Absoluta (FA) e Relativa (FR) e o Valor de Importância de Cobertura das Herbáceas (VICH). Para avaliar a diversidade florística foi utilizado $\mathrm{o}$ índice de Shannon-Wiener ( $\left.\mathrm{H}^{\prime}\right)$, calculado por meio do Software Mata Nativa (CIENTEC, 2002). O Valor de Importância de Cobertura das Herbáceas (VICH) foi determinado somando a Densidade Relativa (DR) e a Frequência Relativa (FR).

Foi elaborada uma matriz de presença/ausência de espécies $\mathrm{x}$ áreas de estudo para verificar as similaridades florísticas entre as áreas de estudo, por análise de agrupamento e da técnica de ligação média de grupo (UPGMA), utilizando o índice de Jaccard. Considerando apenas as listas de espécies, utilizou-se o mesmo índice e o mesmo tipo de agrupamento para comparar as similaridades entre as floras herbáceaarbustivas das áreas estudadas neste trabalho.

\section{Resultados e discussão}

\section{Composição Florística e Densidade do Banco de Sementes do Solo}

No Banco de Sementes do Solo (in situ), nas duas áreas estudadas, foi registrado um total de 2.199 indivíduos, distribuídos em 19 famílias, 54 gêneros e 71 espécies (Tabela $1)$.

$\mathrm{Na}$ análise da Área I foram amostrados
1541 indivíduos, sendo a família Rubiaceae a mais representativa com quatro gêneros e cinco espécies. Entre as espécies de leguminosas coletadas foi possível identificar na Área I a Chamaecrista nictitans com 214 indivíduos distribuídos em 22 unidades amostrais, Aeschynomene denticulata com 2 indivíduos em 2 unidades amostrais, Senna obtusifolia e Chamaecrista desvauxii com 1 indivíduo em 1 unidade amostral. Embora a Chamaecrista nictitans se apresente em maior número de indivíduos, a vagem mede em média $4 \mathrm{~cm}$ de comprimento por $0.5 \mathrm{~cm}$ de largura, o que dificulta a extração e obtenção das sementes.

A Área II apresentou um menor número de espécies de leguminosas, sendo uma única espécie herbáceae trepadeira a $M a$ croptilium lathyroides (Feijão-de-Rolinha). Dentre as forrageiras utilizadas, o feijão-derôla (M. lathyroides (L.) urb.), nativa da América Tropical é uma planta anual que chega a medir até um metro de altura, com crescimento indeterminado (Skerman et al. 1988; Ferreira et al. 2004). Suas flores são vermelho-violáceas ou azul-violáceas com propagação por sementes. Além de sua utilização como forragem, esta planta é empregada na adubação verde, pois participa de interação mutualística para fixação de nitrogênio (Lorenzi, 2000). Esta espécie destaca-se pela sua alta capacidade de formação de biomassa, chegando a atingir $13 \mathrm{t}$ ha-1 de matéria seca quando cultivada em condições ideais, pela elevada produção de sementes de pequeno tamanho, podendo ter de 88.000 a 154.000 sementes por quilograma (FAO 2007) e por grande deiscência de vagens maduras. Pouco exigente em fertilidade, esta planta vegeta em locais mal drenados e com $\mathrm{pH}$ baixo, colonizando áreas com precipitações pluviais anuais entre 430 e $3000 \mathrm{~mm}$. (SKERMAN et al. 1988; FERREIRA et al. 2004).

O banco de sementes possui dinâmica própria, que varia conforme a espécie, condições da semente, ocorrência de predadores e fatores ambientais (Carmona, 1995). O seu tamanho é determinado pela produção 
Rosineide Gonçalves Parente et. al

de sementes, extensão da chuva de sementes, mortalidade de sementes no solo e número de sementes germinadas (Robert, 1981). No estrato herbáceo da caatinga, além destes fatores, tem-se a influência da presença dos animais (caprinos e bovinos) na época de pastejo. Quando este pastejo é feito nas primeiras semanas logo após as chuvas, as herbáceas não conseguem completar seu ciclo de vida, não produzindo sementes, afetando a composição e estrutura do banco de sementes.

Tabela 1. Lista de famílias e espécies ocorrentes nas Áreas I e II, com os respectivos números de indivíduos em cada área de estudo. Área I = Projeto Maria Tereza, Área II = Sítio Asa Branca

$$
\text { FAMÍLIA/ESPÉCIE }
$$

Amaranthaceae

Alternanthera brasiliana (L.) Kuntze

Amaranthus lividus L.

Amaranthus viridis L.

Amaranthus spinosa L.

Froelichia lanata Moq.

Asteraceae

Centratherum punctatum Cass.

Eupatorium sp.

Ageratum conyzoides L.

Acanthospermum hispidum Dc.

Blainvillea latifolia ( L. f.) Dc.

Achyrocline satureioides (Lam.) Dc.

Bidens pilosa $\mathrm{L}$.

Baccharis dracunculifolia Dc.

Bromeliaceae

Bromelia laciniosa Martius ex Schultes f.

Capparaceae

Cleome spinosa Jacq.

Caryophyllaceae

Cerastium glomeratum Thuill.

Cyperaceae

Cyperus sp.

Cyperus diffusus Vahl

Cyperus brevifolius (Rottb.) Hassk.

Euphorbiaceae

Jatropha mollissima Muell. Arg.

Jatropha curcas L.

Croton lundianus (Dierdr.) miill. Arg.

Gramineae

Melinis minutiflora P. beauv.

\section{ÁREA I \\ ÁREA II}

$\begin{array}{ll}50 & - \\ - & 2 \\ - & 2 \\ - & 6 \\ 26 & -\end{array}$

8

50

12

- 3

- 3

- 6

$\begin{array}{ll}- & 2\end{array}$

1

1

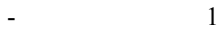

$\begin{array}{ll}- & 2\end{array}$

$\begin{array}{ll}- & 46\end{array}$

$\begin{array}{ll}- & 1\end{array}$

38

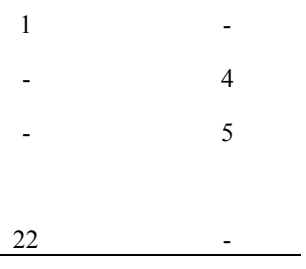

Continua... 
Rosineide Gonçalves Parente et. al

\begin{tabular}{|c|c|c|}
\hline Brachiaria sp. & - & 12 \\
\hline luziola peruviana juss. & - & 52 \\
\hline Paspalum sp. & - & 76 \\
\hline Digitária cilliaris ( Retz.) Koel. & - & 3 \\
\hline Rhynchelytrum repens ( Willd.) C.E. Hubb. & - & 4 \\
\hline Cynodon dactylon ( L.) Pers. & - & 3 \\
\hline Panicum repens $\mathrm{L}$. & - & 13 \\
\hline Chloris polydactyla $(\mathrm{L}$.) Sw. & - & 19 \\
\hline Dactyctenium aegyptium (L.) Beauv. & - & 12 \\
\hline Brachiaria fasciculata ( Sw.) Parodi & - & 10 \\
\hline \multicolumn{3}{|l|}{ Lamiaceae } \\
\hline Marsypianthes chamaedrys(Vahl) Kuntze & 18 & - \\
\hline Marsypianthes chamaedrys(Vahl) Kuntze & - & 31 \\
\hline Hyptis suaveolens (L.) Poit. & - & 7 \\
\hline \multicolumn{3}{|l|}{ Leguminosae } \\
\hline Chamaecrista nictitans Subsp. Pattelaria (collad.) H.S.Irwin \& Barnaby & 214 & - \\
\hline Senna obtusifolia $($ L.) Irwin et Barneby & 1 & - \\
\hline Chamaecrista desvauxii (Collad.) Killip & 1 & - \\
\hline Aeschynomene denticulata Rudd & 2 & - \\
\hline Leguminosa sp. & 9 & - \\
\hline Senna alata (L.) Roxb. & - & 2 \\
\hline Macroptilium lathyroides (L.) Urb. & - & 18 \\
\hline \multicolumn{3}{|l|}{ Loganaceae } \\
\hline Spigelia anthelmia $L$. & - & 7 \\
\hline \multicolumn{3}{|l|}{ Lythraceae } \\
\hline Ammannia coccinea Rottb. & - & 31 \\
\hline \multicolumn{3}{|l|}{ Malvaceae } \\
\hline Pavonia cancellata (L.) Cav. & 15 & - \\
\hline Herissantia crispa (L.) Brizicky & 16 & - \\
\hline Sida glaziovii K. Schum. & 133 & - \\
\hline Sida sp. & 4 & - \\
\hline Sida rhombifolia $L$. & 6 & - \\
\hline Sida spinosa $L$. & - & 19 \\
\hline Sida cordifolia $L$. & - & 53 \\
\hline Pavonia sidifolia Kunth & - & 5 \\
\hline Sida glaziovii K. Schum. & - & 13 \\
\hline Malvastrum americanum (L.) Torr. & - & 1 \\
\hline Sidastrum micranthun (St. Hil.) Fryxell & - & 5 \\
\hline Sida $s p$ & - & 2 \\
\hline
\end{tabular}


Rosineide Gonçalves Parente et. al

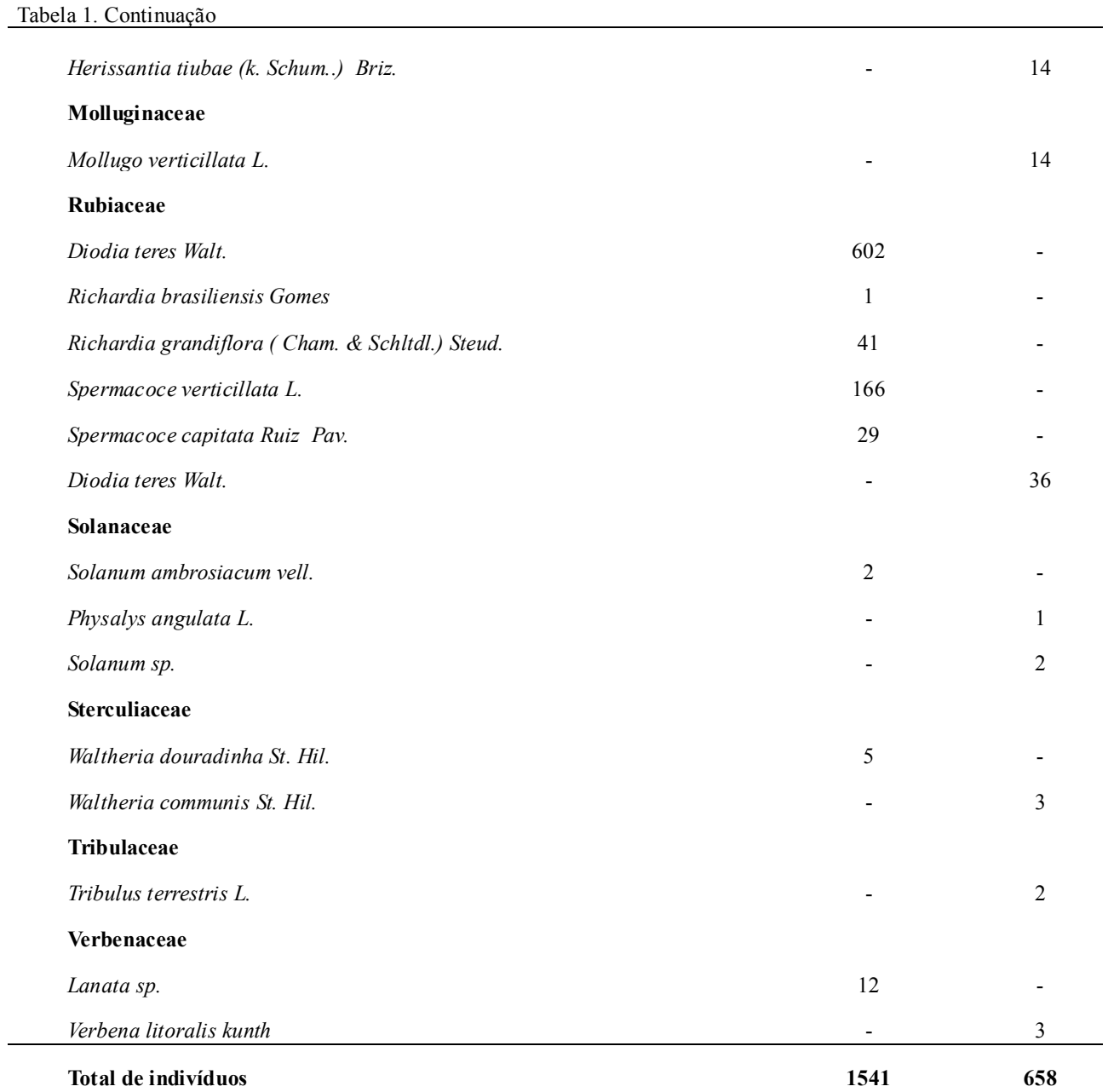

\section{Diversidade Florística}

De acordo com Índice de Diversidade de Shannon-Weaver (H'), a maior diversidade foi encontrada na Área II com 3,23 (Tabela 2), mesmos tendo um menor número de indivíduos (658), comparado com a Área I (1.541). Esse valor pode ser explicado porque o Índice de Diversidade de Shannon-Weaver não avalia apenas a riqueza, mas também a uniformidade de distribuição das espécies no espaço amostral (Tabela 2). Esse fato também se explica pelo caráter dinâmico e heterogêneo das comunidades herbáceas, no que se refere a sua distribuição no espaço e no tempo.

Para o Índice de Uniformidade de Pielou (J), a Área II também apresentou a maior diversidade com 0,83 e 0,63 para a Área I, para esses índices os valores mais próximos de 1 representam maior diversidade.

No BSS in situ, as gramíneas dominaram praticamente a densidade em toda a Área II. Em geral, em Áreas degradadas por atividades agropastoris, que apresentam alta diversidade de gramíneas é difícil o estabelecimento de plântulas originadas das sementes que chegam durante o período seco. Estas pastagens frequentemente são queimadas, eliminando algumas espécies e perpetuando a dominância das gramíneas, que têm capacidade rápida de colonização e crescimento, além de um forte poder competitivo. Isto foi verificado nas regiões áridas no leste da África por Duncan et al. (1999). 
Rosineide Gonçalves Parente et. al

Tabela 2. Diversidade de espécies herbáceas em áreas de caatinga. Área I = Projeto Maria Tereza; Área II. Projeto N6-Sítio Asa Branca; $\mathrm{N}$ = números de indivíduos; $\mathrm{S}=$ Número de espécies; $\ln (\mathrm{s})=$ Diversidade. Máxima, H' = Índice de Diversidade de Shannon-Wiener; $\mathrm{C}=$ Índice de Uniformidade de Pielou; $\mathrm{J}=$ Índice de Simpson e QM $=\mathrm{Coeficiente}$ de Mistura de Jentsch.

\begin{tabular}{cccccccc}
\hline BSS IN SITU & $\mathbf{N}$ & $\mathbf{S}$ & $\mathbf{I n}(\mathbf{S})$ & $\mathbf{H}^{\prime}$ & $\mathbf{C}$ & $\mathbf{J}$ & QM \\
\hline ÁREA I & 1541 & 42 & 3,74 & 2,36 & 0,81 & 0,63 & $01: 36,7$ \\
ÁREA II & 658 & 49 & 3,89 & 3,23 & 0,94 & 0,83 & $01: 13,4$ \\
TOTAL DE INDIVÍDUOS & 2199 & & & & & & \\
\hline
\end{tabular}

\section{Análise Fitossociológica da Área I}

$\mathrm{Na}$ avaliação comparativa dos parâmetros fitossociológicos dessa área, verifica-se na tabela 3 que, a espécie Diodia teres obteve a maior densidade relativa (DR) e valor de importância (VI), cujos valores foram 39,58 e 62,4 respectivamente, seguida pelas espécies Spermacoce verticillata L com 10,9 (DR) e 19,154 (VI), Chamaecrista nictitans Subsp. Pattelaria (collad.) H.S.Irwin \& Barnaby com 8,96 (DR) e 13,324 (VI) e Sida glaziovii K. Schum com 7,07 (DR) e 18,238 (VI). O somatório do número de indivíduos dessas espécies é igual a 1.025, representando $66,5 \%$ total de indivíduos. Todos os indivíduos das demais espécies somadas são iguais a 516, representando $33,5 \%$ do total de indivíduos.

Em relação à frequência a espécie que apresentou a melhor distribuição foi a Diodia teres Walt e a Sida glaziovii com os valores de 47 e 23 para Frequência Absoluta (FA) e 22,82 e 11,17 para Frequência Relativa (FR) respectivamente (Tabela 3 ).

Tabela 3. Parâmetros fitossociológicos das espécies com maiores Valores de Importância de Cobertura das Herbáceas (VICH) nos Bancos de Sementes do Solo in situ para a Área I. Densidade Relativa (DR); Freqüência Absoluta (FA); Freqüência Relativa (FR), Número de Indivíduos (N).

\begin{tabular}{|c|c|c|c|c|c|c|}
\hline Nome Científico & $\mathbf{N}$ & DR & FA & FR & VICH & VICH (\%) \\
\hline Diodia teres & 610 & 39,58 & 47 & 22,82 & 39,585 & 19,79 \\
\hline Spermacoce verticillata & 168 & 10,9 & 17 & 8,25 & 10,902 & 5,45 \\
\hline Sida glaziovii & 109 & 7,07 & 23 & 11,17 & 7,073 & 3,54 \\
\hline Chamaecrista nictitans & 138 & 8,96 & 9 & 4,37 & 8,955 & 4,48 \\
\hline Alternanthera brasiliana & 85 & 5,52 & 10 & 4,85 & 5,516 & 2,76 \\
\hline Chamecrista nictitans & 49 & 3,18 & 9 & 4,37 & 3,18 & 1,59 \\
\hline Spermacoce capitata & 31 & 2,01 & 7 & 3,4 & 2,012 & 1,01 \\
\hline Eupatorium sp. & 28 & 1,82 & 5 & 2,43 & 1,817 & 0,91 \\
\hline Richardia grandiflora & 40 & 2,6 & 3 & 1,46 & 2,596 & 1,3 \\
\hline Marsypianthes chamaedrys & 20 & 1,3 & 5 & 2,43 & 1,298 & 0,65 \\
\hline Chamecrista nictitans & 27 & 1,75 & 4 & 1,94 & 1,752 & 0,88 \\
\hline Pavonia cancellata & 10 & 0,65 & 6 & 2,91 & 0,649 & 0,32 \\
\hline Leguminosa 01 & 8 & 0,52 & 6 & 2,91 & 0,519 & 0,26 \\
\hline Froelichia lanata & 27 & 1,75 & 3 & 1,46 & 1,752 & 0,88 \\
\hline Eupatorium sp. & 24 & 1,56 & 3 & 1,46 & 1,557 & 0,78 \\
\hline Lantana sp. & 12 & 0,78 & 4 & 1,94 & 0,779 & 0,39 \\
\hline Centraterum punctatum & 8 & 0,52 & 4 & 1,94 & 0,519 & 0,26 \\
\hline Pavonia cancellata & 5 & 0,32 & 4 & 1,94 & 0,324 & 0,16 \\
\hline Herissantia crispa & 16 & 1,04 & 2 & 0,97 & 1,038 & 0,52 \\
\hline Melinis minutiflora & 22 & 1,43 & 1 & 0,49 & 1,428 & 0,71 \\
\hline Ageratum conyzoides & 12 & 0,78 & 1 & 0,49 & 0,779 & 0,39 \\
\hline Richardia brasiliensis & 3 & 0,19 & 2 & 0,97 & 0,195 & 0,1 \\
\hline Wattheria douradinha & 2 & 0,13 & 2 & 0,97 & 0,13 & 0,06 \\
\hline Desconhecida 01 & 2 & 0,13 & 2 & 0,97 & 0,13 & 0,06 \\
\hline Solanum ambrosiacum & 5 & 0,32 & 1 & 0,49 & 0,324 & 0,16 \\
\hline Sida rhombifolia & 5 & 0,32 & 1 & 0,49 & 0,324 & 0,16 \\
\hline Sida $s p$ & 4 & 0,26 & 1 & 0,49 & 0,26 & 0,13 \\
\hline \multirow[t]{2}{*}{ Alternanthera brasiliana } & 3 & 0,19 & 1 & 0,49 & 0,195 & 0,1 \\
\hline & & & & & & Continua... \\
\hline
\end{tabular}


Rosineide Gonçalves Parente et. al

\begin{tabular}{|c|c|c|c|c|c|c|}
\hline Tabela 3. Continuação & & & & & & \\
\hline Watheria douradinha & 2 & 0,13 & 1 & 0,49 & 0,13 & 0,06 \\
\hline Aeschynomene sp. & 1 & 0,06 & 1 & 0,49 & 0,065 & 0,03 \\
\hline Bromelia laciniosa & 1 & 0,06 & 1 & 0,49 & 0,065 & 0,03 \\
\hline Jatropha mollissima & 1 & 0,06 & 1 & 0,49 & 0,065 & 0,03 \\
\hline Mimosa sp. & 1 & 0,06 & 1 & 0,49 & 0,065 & 0,03 \\
\hline Sida rhombifolia & 1 & 0,06 & 1 & 0,49 & 0,065 & 0,03 \\
\hline Solanum ambrosiacum & 1 & 0,06 & 1 & 0,49 & 0,065 & 0,03 \\
\hline Waltheria douradinha & 1 & 0,06 & 1 & 0,49 & 0,065 & 0,03 \\
\hline Senna obtusifolia & 1 & 0,06 & 1 & 0,49 & 0,065 & 0,03 \\
\hline Chamaecrista desvauxii & 1 & 0,06 & 1 & 0,49 & 0,065 & 0,03 \\
\hline Solanum ambrosiacum Vell. & 1 & 0,06 & 1 & 0,49 & 0,065 & 0,03 \\
\hline Marsypianthes chamaedrys & 1 & 0,06 & 1 & 0,49 & 0,065 & 0,03 \\
\hline Aeschynomene sp. & 1 & 0,06 & 1 & 0,49 & 0,065 & 0,03 \\
\hline
\end{tabular}

\section{Análise Fitossociológica da Área II}

$\mathrm{Na}$ avaliação dessa área, verificou-se que as espécies Paspalum sp., Sida cordifolia L., Luziola peruviana juss., Diodia teres Walt, Marsypianthes chamaedrys (Vahl) Kuntze, Cyperus brevifolius (Rottb.) Hassk., Ammannia coccinea Rottb. e Cyperus sp. apresentaram os maiores valores de Densidade Relativa (DR) e Valor de Importância de Cobertura das Herbáceas (VICH). O soma do número de indivíduos dessas es- pécies é igual a 383, representando 58,2\% do total. Todos os indivíduos das demais espécies somadas são iguais a 275 , representando $41,8 \%$ do total. Essa área apresentou uma alta densidade de gramíneas o que pode evidenciar o uso com atividades agrícolas e agropastoris. Essas espécies também obtiveram os maiores valores de frequência absoluta e relativa, exceto a espécie Cyperus sp. com 2 e 1.19 para frequência absoluta e relativa, respectivamente, como pode ser visto na tabela 4.

Tabela 4 -. Parâmetros fitossociológicos das espécies com maiores Valores de Importância de Cobertura das Herbáceas (VICH) nos Bancos de Sementes do Solo in situ para a Área II. Densidade Relativa (DR); Frequência Absoluta (FA); Frequência Relativa (FR), Número de Indivíduos (N).

\begin{tabular}{|c|c|c|c|c|c|c|}
\hline Nome Científico & $\mathbf{N}$ & DR & FA & FR & VICH & VICH $(\%)$ \\
\hline Paspalum sp. & 88 & 13,37 & 12 & 7,14 & 13,374 & 6,69 \\
\hline Sida cordifolia L. & 48 & 7,29 & 21 & 12,5 & 7,295 & 3,65 \\
\hline Luziola peruviana juss. & 57 & 8,66 & 12 & 7,14 & 8,663 & 4,33 \\
\hline Diodia teres Walt. & 39 & 5,93 & 13 & 7,74 & 5,927 & 2,96 \\
\hline Marsypianthes chamaedrys(Vahl) Kuntze & 36 & 5,47 & 11 & 6,55 & 5,471 & 2,74 \\
\hline Cyperus brevifolius (Rottb.) Hassk. & 38 & 5,78 & 9 & 5,36 & 5,775 & 2,89 \\
\hline Ammannia coccinea Rottb. & 31 & 4,71 & 7 & 4,17 & 4,711 & 2,36 \\
\hline Cyperus sp. & 46 & 6,99 & 2 & 1,19 & 6,991 & 3,5 \\
\hline Sida Spinosa L. & 19 & 2,89 & 5 & 2,98 & 2,888 & 1,44 \\
\hline Sida glaziovii K. Schum. & 13 & 1,98 & 5 & 2,98 & 1,976 & 0,99 \\
\hline Paspalum sp. & 17 & 2,58 & 4 & 2,38 & 2,584 & 1,29 \\
\hline Jatropha curcas L. & 8 & 1,22 & 6 & 3,57 & 1,216 & 0,61 \\
\hline Dactyctenium aegyptium (L.) Beauv. & 14 & 2,13 & 4 & 2,38 & 2,128 & 1,06 \\
\hline Chloris polydactyla (L.) Sw. & 19 & 2,89 & 2 & 1,19 & 2,888 & 1,44 \\
\hline Mollugo verticillata L. & 14 & 2,13 & 3 & 1,79 & 2,128 & 1,06 \\
\hline Herissantia tiubae (k. Schum..) Briz. & 9 & 1,37 & 4 & 2,38 & 1,368 & 0,68 \\
\hline Panicum repens L. & 12 & 1,82 & 2 & 1,19 & 1,824 & 0,91 \\
\hline Sidastrum micranthun (St. Hil.) Fryxell & 8 & 1,22 & 3 & 1,79 & 1,216 & 0,61 \\
\hline Hyptis suaveolens (L.) Poit. & 7 & 1,06 & 3 & 1,79 & 1,064 & 0,53 \\
\hline Achyrocline satureioides (Lam.) Dc. & 6 & 0,91 & 3 & 1,79 & 0,912 & 0,46 \\
\hline Brachiaria sp. & 12 & 1,82 & 1 & 0,6 & 1,824 & 0,91 \\
\hline
\end{tabular}


Rosineide Gonçalves Parente et. al

\begin{tabular}{|c|c|c|c|c|c|c|}
\hline Spigelia anthelmia L. & 7 & 1,06 & 2 & 1,19 & 1,064 & 0,53 \\
\hline Brachiaria fasciculata ( Sw.) Parodi & 10 & 1,52 & 1 & 0,6 & 1,52 & 0,76 \\
\hline Croton lundianus (Dierdr.) miill. Arg. & 5 & 0,76 & 2 & 1,19 & 0,76 & 0,38 \\
\hline Waltheria communis St. Hil. & 3 & 0,46 & 2 & 1,19 & 0,456 & 0,23 \\
\hline Amaranthus spinosa L. & 6 & 0,91 & 1 & 0,6 & 0,912 & 0,46 \\
\hline Senna alata (L.) Roxb. & 2 & 0,3 & 2 & 1,19 & 0,304 & 0,15 \\
\hline Sida sp. & 2 & 0,3 & 2 & 1,19 & 0,304 & 0,15 \\
\hline Tribulus terrestris $\mathrm{L}$. & 2 & 0,3 & 2 & 1,19 & 0,304 & 0,15 \\
\hline Pavonia sidifolia Kunth & 5 & 0,76 & 1 & 0,6 & 0,76 & 0,38 \\
\hline Herissantia tiubae ( k. Schum.) Briz. & 5 & 0,76 & 1 & 0,6 & 0,76 & 0,38 \\
\hline Rhynchelytrum repens ( Willd.) C.E. Hubb. & 4 & 0,61 & 1 & 0,6 & 0,608 & 0,3 \\
\hline Acanthospermum hispidum Dc. & 3 & 0,46 & 1 & 0,6 & 0,456 & 0,23 \\
\hline Blainvillea latifolia ( L. f.) Dc. & 3 & 0,46 & 1 & 0,6 & 0,456 & 0,23 \\
\hline Cynodon dactylon ( L.) Pers. & 3 & 0,46 & 1 & 0,6 & 0,456 & 0,23 \\
\hline Digitaria cilliaris ( Retz.) Koel. & 3 & 0,46 & 1 & 0,6 & 0,456 & 0,23 \\
\hline Verbena litoralis kunth & 3 & 0,46 & 1 & 0,6 & 0,456 & 0,23 \\
\hline Amaranthus lividus L. & 2 & 0,3 & 1 & 0,6 & 0,304 & 0,15 \\
\hline Amaranthus viridis L. & 2 & 0,3 & 1 & 0,6 & 0,304 & 0,15 \\
\hline Bidens pilosa $\mathrm{L}$. & 2 & 0,3 & 1 & 0,6 & 0,304 & 0,15 \\
\hline Cerastium glomeratum Thuill. & 2 & 0,3 & 1 & 0,6 & 0,304 & 0,15 \\
\hline Baccharis dracunculifolia Dc. & 1 & 0,15 & 1 & 0,6 & 0,152 & 0,08 \\
\hline Cleome spinosa Jacq. & 1 & 0,15 & 1 & 0,6 & 0,152 & 0,08 \\
\hline Cyperus diffusus Vahl & 1 & 0,15 & 1 & 0,6 & 0,152 & 0,08 \\
\hline Malvastrum americanum (L.) Torr. & 1 & 0,15 & 1 & 0,6 & 0,152 & 0,08 \\
\hline Physalis angulata $\mathrm{L}$. & 1 & 0,15 & 1 & 0,6 & 0,152 & 0,08 \\
\hline Solanum sp. & 1 & 0,15 & 1 & 0,6 & 0,152 & 0,08 \\
\hline Panicum repens L. & 1 & 0,15 & 1 & 0,6 & 0,152 & 0,08 \\
\hline Total de indivíduos & $\begin{array}{r}65 \\
8\end{array}$ & 100 & 168 & 100 & 100 & 100 \\
\hline
\end{tabular}

\section{Conclusões}

O estrato herbáceo da caatinga é formado por espécies subarbustivas e herbáceas anuais, ruderais, invasoras, com ciclo biológico curto. Possui uma grande diversidade de espécies, muito importante para as comunidades.

O ciclo das espécies que compõem o estrato herbáceo da caatinga é efêmero, se inicia no período das chuvas. As sementes produzidas por este estrato que sobrevive até a estação chuvosa dão origem à próxima geração. Como estratégia de sobrevivência, independendo das variações ambientais, nem todas as sementes germinam. Assim, em cada ano, a população fica determinada, não só pelas sementes do ano anterior, mas também pelas sementes que germinaram naquele ano.
A composição e tamanho de um banco de sementes são determinados pela produção, chuva de sementes, mortalidade e pelo número de sementes germinadas. A atuação de animais nas áreas estudadas, mascara a composição deste estrato, sendo necessário desenvolver outros estudos, particularmente em parcelas protegidas contra a herbívoria, o que permitirá detectar sua densidade e diversidade.

\section{Referências}

ALCOFORADO-FILHO, F. G. et al. Florística e fitossociologia de um remanescente de vegetação caducifólia espinhosa arbórea em Caruaru, Pernambuco. Acta Bot. Bras. v. 17. n. 2. p. 287-303. 2003. 
Rosineide Gonçalves Parente et. al

ARAÚJO, F. S. et al. Composição florística da vegetação de carrasco, Novo Oriente, CE. Revista Brasileira de Botânica. v. 21, n. 2. p. 105-116. 1998.

ARAÚJO, M. M. et al. Densidade e composição florística do banco de sementes do solo de florestas sucessionais na região do Baixo Rio Guamá, Amazônia Oriental, Scientia Forestalis, v. 59, p. 115-130. 2001.

ARAÚJO, E. L. et al. Diversidade de herbáceas em microhabitats rochoso, plano e ciliar em uma área de caatinga, Caruaru, PE, Brasil. Acta. Bot. Bras. v. 19, n. 2, p. 287-296. 2005.

BAIDER, C., O banco de sementes e de plântulas na sucessão da Mata Atlântica. 98p., 1994. Dissertação (Mestrado em Ciências). Universidade de São Paulo, São Paulo.

BAIDER, C.; TABARELLI, M; MANTOVANI, W, O Banco de sementes de um trecho de uma floresta Atlântica montana (São Paulo - Brasil). Rev. Bras. Biol. v. 59, n. 2, p. 319-328. 1999.

BAKER, H. G., Some aspects of the natural history of seed banks. 1989.

BANDEL, G. Chromosome numbers and evolution in the Leguminosae. Caryologia. v. 27, p. 17-32. 1974.

BARBOSA, M. R. V., Estudo florístico e fitossociológico da Mata do Buraquinho, remanescente de Mata Atlântica em João Pessoa, PB. 1996. Tese (Doutorado em Ciências). Universidade Estadual de Campinas, Campinas, SP.

BARRALIS, G.; CHADOEUF, R., LOCHAMP, J. P., Longeté des semences de mauvaises herbes annuelles dans un sol cultivé. Weed Research. v. 21, n. 6, p. 407-418. 1988.

BATALHA, M. A.; MANTOVANI, W., Reproductive phenological patterns of cerrado plant species at the Pé-de-Gigante Reserve
(Santa Rita do Passa Quatro, SP, Brazil): a comparison between the herbaceous and woody floras. Rev. Bras. Biol. v. 60, n. 1, p. 129-145. 2000.

BELTRÃO, G. T. A.; GUERRA, M. Citogenética de angiospermas coletadas em Pernambuco - III. Ci. e Cult. v. 42., p. 839-845. 1990.

BLUMENSCHEIN, A. Número de cromossomas de algumas espécies de orquídeas. Publicação científica v. 1, p. 45-50. 1960.

CAETANO, R. S. X.; CHRISTOFFOLETI, P. J.; VICTORIA FILHO, R., "Banco" de sementes de plantas daninhas em pomar de laranjeira 'pera'. Sci. agric. v. 58, n. 3, p. 509517. 2001.

CAETANO, R. S., Dinâmica do banco de sementes de plantas daninhas na cultura dos citros (Citrus sinensis (L.) Osbeck) submetida a diferentes sistemas de manejo. Piracicaba. 2000. 105p. Tese (Doutorado em Agronomia). Universidade de São Paulo, São Paulo

CARVALHEIRA,,G. M. G. et al. Citogenética de angiospermas coletadas em Pernambuco IV. Acta Bot. Bras. v. 5, n. 2, p. 37-51. 1991.

CESTARO, L. A., WAECHTER, J. L.; BAPTISTA, L. R. M., Fitossociologia do estrato herbáceo da mata de araucária da Estação Ecológica de Aracuri, Esmeralda, RS. Hoehnea. v. 13, p.59-72. 1986.

CHRISTOFFOLETI, P. J.; CAETANO, R. S. $\mathrm{X}$., Banco de sementes do solo. Sci. agric. v. 55, p. 74-78. 1998.

CITADINI-ZANETTE, V., Composição florística e fitossociologia da vegetação herbácea terrícola de uma mata de Torres, Rio Grande do Sul, Brasil. Iheringia. v. 32, p. 2362. 1984.

CITADINI-ZANETTE, V.; BAPTISTA, L. R. M., Vegetação herbácea terrícola de uma comunidade florestal em Limoeiro, município 
Rosineide Gonçalves Parente et. al

de Torres, Rio Grande do Sul, Brasil. Boletim do Instituto de Biociências da UFRGS. v. 45, p.1-87. 1989.

COSTA, R. C.; ARAÚJO, F. S., Densidade, germinação e flora do banco de sementes no solo, no final da estação seca, em uma área de caatinga, Quixadá, CE. Acta Bot. Bras. v. 17, n. 2, p. 259-264. 2003.

DALLING, J. W.; SWAINE, M. D.; GARWOOD, N. C. Soil seed bank community dynamics in seasonally moist lowland tropical forest, Panama. Journald of Tropical Ecology, v. 13, p. 659-680, 1997.

DESSAINT, F.; CHADOEUF, R.; BARRALIS, G., Estude de la dynamique communauté adventice: III. Influence à long terme dês techniques culturales sur la composition spécifique du stock semencier. Weed Research. Oxford. v. 30, p. 319-330. 1990.

DIESEL, S., Estudo fitossociológico herbáceo/arbustivo da mata ripária da bacia hidrográfica do Rio dos Sinos, RS. Pesquisas Série Botânica. v. 42, p. 201-257. 1991.

DORNELES, L. P. P.; NEGRELE, R. R. B., Composição florística e estrutura do compartimento herbáceo de um estágio sucessional avançado da Floresta Atlântica, no sul do Brasil. Biotemas. v. 12, p. 7-30. 1999.

EHRENDORFER, F. Polyploid and distribution. In: Polyploid - Biological relevance (Lewis, W. H., ed.) Plenum Press, New York, p. 45-60. 1980.

FEDEROV, Am. A. (ed.). Chomosome numberos flowering plants. Komarov Botanical Institute, Leningrad. 1969.

FELIX, L. P. Citogenética e citotaxonomia de orquídeas do Brasil. (Tese de doutorado em Botânica). 2001. Universidade Federal Rural de Pernambuco.

FERNÁNDEZ-QUINTANILLA, C. Studyng the population dynamics of weeds. Weed
Research. v. 25, n. 6, p. 443-447. 1988.

FERNANDEZ-QUINTANILLA, C.; SAAVEDRA, M. S; GARCIA TORRE, L., Ecologia de las malas hierbas. In: GARCIA TORRE, L.; FERNANDEZ- QUINTANILLA, C. Fundamentos sobre malas hierbas y herbicidas. Mundi-Prensa. Madrid. p. 49-69. 1991.

FERRAZ, E. M. M. et al. Composição florística em trechos de vegetação de caatinga e brejo de altitude na região do vale do Pajeú, Pernambuco. Revista Brasileira de Botânica v. 21, n. 1, p. 7-15. 1998.

GARWOOD, N. C., Tropical soil seed banks: a review In: M. A. Leck, V. T. Parker e R. L. Simpson (eds.), Ecology of soil seed banks. San Diego, Academic Press. p. 149-209. 1989.

GOMES, M. A. F. Padrões de caatinga nos Cariris Velhos, Paraíba. 145p. 1979. (Dissertação de Mestrado). Universidade Federal Rural de Pernambuco, Recife.

GRANT, V. Especiación Vegetal. In: Noriega Editores, Mexico. 587p. 1989

GUERRA, M. S., Introdução á Citogenética Vegetal. Rio de Janeiro. 1988a. 141p.

GUERRA, M. Characterization of different types of condesed chromatin in Costas (Zingiberaceae). PI. Syst. Evol. v. 158, p. 107115. 1988b.

GUERRA, M. Mitotic and meiotic analysis of a pericentric invertion associated with a duplication in Eleutiierine bulbosa.

Chromosoma. v. 97, p. 80-87. 1988c.

GUERRA, M. Cis-acting regulation of NOR cistron in Eleutherine bulbosa (Iridaceae). Genética v. 83, p. 235-241. 1991.

GUERRA, M. Citogenética de angiospermas coletadas em Pernambuco -1. Rev. Brasil. Genet. v. 9, p. 21-40. 1986. 
Rosineide Gonçalves Parente et. al

GUERRA, M.; NOGUEIRA, M. T. M. The cytotaxonomy of Emilia ssp. (Asteraceae: Senecioneae) ocurring in Brazil. PI. Syst. Evol. v. 170, p. 229-236. 1990.

GUERRA, M. Chromosome number variation and evolution in monocots. In: Wilson, K. L. e Morrison, D. A. (eds), Monocots II Systematics and Evolution. CSIRO Publ., Collingwood. pp. 127-136. 2000.

GUERRA, M.; SOUZA, M. J. Como observer cromossomos: um guia de técnicas em citogenética vegetal, animal e humana. Ribeirão Preto, SP. 2002, 131p.

HEYWOOD, V. H. Taxonomia Vegetal. Edward Arnold. London. 1970. 200p.

HOPKINS, M. S.; GRAHAM, A. W.,. The composition of soil seed banks beneath lowland tropical rainforests in North Queensland, Australia. Biotropica, v. 15, p. 90-99. 1983.

JONES, K; JOPLING, C., Chromosomes and classification of the Commelinaceae. Bot. J. Linn. Soc. v. 65,p. 129-162. 1972.

LIMA, P. C. F.; OLIVEIRA, E. B. de; MACHADO, S. do A., Equações para estimativa de biomassa de espécie de Prosopis no semi-árido brasileiro. Boletim de Pesquisa Florestal. v. 32/33, p. 67-70. 1996.

LIMA, P. F. L. Comportamento silvicultural de espécies de Prosopis, em Petrolina-PE, região semi-árida brasileira. 1994. 110p. (Tese de Doutorado). Universidade Federal do Paraná. Curitiba, Paraná, PR.

LIMA, K. A.; Caracterização florística e fitossociológica do componente herbáceo ocorrente em áreas da caatinga do cristalino e sedimentar no município de Petrolândia, PE. 2004, 80p. Dissertação (Mestrado em Botânica), Universidade Federal Rural de Pernambuco, Pernambuco, PE.

MANTOVANI, W., Análise florística e fitossociológica do estrato herbáceo- subarbustivo do cerrado da Reserva Biológica de Moji-Guaçu e em Itirapina, São Paulo. 203p. 1987. (Tese de Doutorado). Universidade Estadual de Campinas. Campinas, São Paulo, SP.

MARCHEZAN, E.; CORADINI, J., Z.; AVILA, L. A.; SEGABINAZZI, T. Eficiência da avaliação do banco de sementes na predição da infestação por arroz vermelho e rendimento de grãos do arroz irrigado após dois anos de rotação de cultura e pousio do solo. Rev. Bras. de agrociência. v. 7, n. 1, p. 15-17 . 2001.

MELO et al. Karyology and cytotaxomy of the genus Passiflora L. (Passifloraceae). Plant. Syst. Evol. v. 226, p. 69-84. 2001

MORAWETZ, W. Remarks on karyological differentiation patterns in tropical woody plants. PI. Syst. Evol. v. 152, p. 49-100. 1986.

MULLER, S. C.; WAECHTER, J. L., Estrutura sinusial dos componentes herbáceo e arbustivo de uma floresta costeira subtropical. Revista Brasileira de Botânica. V. 24, n.4, p. 395-406. 2001.

OLIVEIRA, V. R. de Diversidade genética em populações de algaroba (Prosopis juliflora (Sw.) D.C.) na região semi-árida do Nordeste Brasileiro. 1999. Tese de Doutorado. Universidade de São Paulo, Piracicaba, SP.

PEDROSA, A. et al. Chromosomal map of the model Legume Lotus japonicus. Genetics. v. 161, p. 1661-1672. 2002.

PEREIRA, I. M.; ANDRADE, L. A. de; BARBOSA, M. R. de V., Composição florística e análise fitossociológica do componente arbustivo-arbóreo de um remanescente florestal no Agreste Paraibano. Acta Bot. Brás. v. 16, n. 3, p. 357-369. 2002.

PITREZ, R. S. Citogenética de representantes da família Commelinaceae do Nordeste do Brasil. 1988. Dissertação (Mestrado em Botânica). Universidade Federal Rural de Pernambuco, Recife, PE. 
Rosineide Gonçalves Parente et. al

PUTZ, F. E.; APPANAH, S., Buried seeds, newly dispersed seeds, and dynamics of a lowland forest in Malaysia. Biotropica. v. 19, p. 326-333, 1987.

RAMALHO, F. C. Taxonomia e número cromossômico de representantes da família Araceae em Pernambuco. 1994. 254p. Dissertação (Mestrado em Botânica). Universidade Federal Rural de Pernambuco, Recife, PE.

RICE, K.J., Impacts of seed banks on grassland community structure and population dynamics. In: LECK, M.A.; PARKER, V.T.; SIMPSON, R.L. (eds.). Ecology of soil seed banks. London: Academic Press, p. 69-86. 1989.

REIS, A. M. S. Organização do estrato herbáceo em uma área de caatinga de Pernambuco em anos consecutivos. 2003. 55p. Dissertação (Mestrado em Botânica Vegetal). Universidade Federal Rural de Pernambuco, Recife, PE.

ROBERTS, H.A., Seed banks in the soil. Advances in Applied Biology. v. 6, p. 1-55. 1981.

RODAL, M. J. N. F; SAMPAIO, E. V. S. B.; FIGUEIREDO, M. A., Manual sobre métodos de estudos florísticos e fitossociológicos ecossistema caatinga. Sociedade Botânica do Brasil, Brasília. 1992.

RODAL, M. J. N. et al. Fitossociologia do componente lenhoso de um refúgio vegetacional no município de Buíque, Pernambuco. Revista Brasileira de Biologia v. 58, n. 3, p. 517-526. 1998.

RODAL, M. J. N.; NASCIMENTO, L. M. do ; MELO, A. L. de, Composição florística de um trecho de vegetação arbustiva caducifólia no município de Ibimirim, PE, Brasil. Acta Botanica Brasilica v. 13, n. 1, p. 15-28. 1999.

RODRIGUES, S. M. C. B., Florística e fitossociologia de uma área de cerrado em processo de desertificação no município de Guilbués - PI. 138p. 1998. (Dissertação de mestrado em Botânica). Universidade Federal Rural de Pernambuco, Recife, PE.

SÀ, C. F. C., Regeneração em área de floresta de restinga na Reserva Ecológica Estadual de Jacarepiá, Saquarema/Rj: I - estrato herbáceo. Arquivos do Jardim Botânico do Rio de Janeiro. v. 34, n. 1, p. 177-192. 1996.

SAMBUICHI, R. H. R., Fitossociologia e diversidade de espécies arbóreas em Cabruca (Mata atlântica raleada sobre plantação de cacau) região Sul da Bahia, Brasil. Acta Botânica Brasílica. v. 16, n. 1, p. 89-101. 2001.

SEVERINO, F. J. e CHRISTOFFOLETI, P. J., Banco de sementes de plantas daninhas em solo cultivado com adubos verdes. Bragantia. v. 60 , n. 3, p. 201-204. 2001.

SIMPSON, R. L.; LECK, M. A.; PARKER, V. T., Seed banks: General concepts and methodological issues. In: LECK, M. A.; PARKER, V. T; SIMPSON, R.L. (Ed). Ecology of soil seed banks. London: Academic Press. p. 3-8. 1989.

SOARES, M. M. M.; GUERRA, M. S.; GALLINDO, F. Citogenética de angiospermas coletadas em Pernambuco - II. Ciência e Cultura. v. 40, n. 8, p. 780-786. 1988.

SOUZA, A. C. R., Levantamento florístico do sub-bosque de um fragmento de floresta atlântica, Recife-PE. 2000. 115 p. Dissertação (Mestrado em Botânica). Universidade Federal Rural de Pernambuco. Recife, PE.

STEBBINS, G. L. Chromosome variation and evolution. Science. v. 152, p. 1463-1469. 1971.

TEMPLETON, A. R.; LEVIN, D. A., Evolutionary consequences of seed pools. American Naturalist, Chicago. v. 114, p. 232249. 1979. 
Rosineide Gonçalves Parente et. al

VANZELA, A. L .L. Localization of 45S rDNA and telomeric sites on holocentric chromosomes of Rhynchospora tenuis Link (Cyperaceae). Genetic and Molecular Biology. v. 26, p.199-201. 2003.

VÁZQUEZ-YANES, C.; OROZCOSEGOVIA, A., Fisiologia ecologica de semillas en la Estacion de Biologia Tropical "Los Tuxtlas", Veracruz, Mexico. Rev. Biol. Trop. v. 35 (suplemento 1), p. 85-89. 1987.

VICENTE, A., Levantamento florístico de um fragmento florestal na Serra de ItabaianaSergipe. 1999. 113p. Dissertação (Mestrado em Botânica), Universidade Federal Rural de Pernambuco, Recife, PE.
VIEIRA, C. M.; PESSOA, S. V. A., Estrutura e composição florística de estrato herbáceosubarbustivo de um pasto abandonado na Reserva Biológica de Poços das Antas, município de Silva Jardim, RJ. Rodriguésia. v. 52, n. 80, p. 17-30. 2001.

WHITMORE, T. C., An introduction to tropical rain forests, Blackwell, London. 1990.

ZICKEL, C. S., Fitossociologia e dinâmica do estrato herbáceo de dois fragmentos do Estado de São Paulo. 1995. 125p. Tese (Doutorado em Engenharia Florestal). Universidade de Campinas. Campinas, SP. 